\title{
Novel gut microbiota-derived metabolite promotes platelet thrombosis via adrenergic receptor signalling
}

Higher PAGIn
levels were
independently
linked with
incident MACE
risk

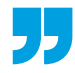

Phenylacetylglutamine (PAGln) is a gut microbial metabolite that can promote platelet hyper-reactivity and increase platelet thrombotic potential via adrenergic receptors to increase the risk of cardiovascular events. This metabolite, discovered using untargeted metabolomics, was first described by Stanley Hazen and colleagues in a study published in Cell.

Untargeted metabolomics, which involves global quantitative analysis of the metabolome, has emerged as a useful tool for the discovery of mechanistic pathways linked to disease.

"[Using this platform], we discovered a chemical signature in blood known as PAGln that was elevated in patients with diabetes mellitus and tracked with cardiovascular disease risks," explains Hazen, the lead investigator of the study. In a cohort of 4,000 patients undergoing elective diagnostic cardiac evaluation, higher levels of PAGln in the plasma were observed in patients with type 2 diabetes as well as in patients with major adverse cardiovascular events (MACE). Higher PAGln levels were independently linked with incident MACE risk, even after adjusting for traditional cardiovascular risk factors. PAGln production was subsequently found to be a gut microbiota-dependent process in both mice and humans.

The investigators then sought to determine the pathophysiological mechanisms underlying the observed relationship between PAGln levels and thrombotic events in humans. PAGln modulated platelet function by promoting platelet adhesion to collagen surfaces in whole blood under physiological shear stress conditions and by inducing an increase in platelet stimulus-dependent $\mathrm{Ca}^{2+}$ release and responsiveness. Furthermore, mice treated with PAGln had an increase in platelet thrombus formation within the injured carotid artery and a reduction in the rate of cessation of blood flow after injury compared with vehicle-treated mice.

To determine whether the genes involved in PAGln generation could regulate platelet function and thrombosis potential, the investigators performed functional and genetic engineering studies with human commensal bacteria. The gut microbial genes por A (involved in oxidative metabolism of phenylalanine, a precursor of PAGln) and fldH (involved in the catalytic reductive metabolism of phenylalanine) were shown to modulate host thrombosis potential in vivo.

Next, the molecular pathways involved in PAGln-induced cellular effects were assessed. Using label-free dynamic mass redistribution (DMR), an assay that enables real-time detection of integrated cellular responses in living cells, PAGln was found to have receptor-ligand interaction properties with cells. By contrast, phenylalanine, a structural analogue of PAGln, does not bind to a cell receptor and therefore showed no DMR response. The PAGln-mediated DMR response in human bone marrow-derived megakaryoblast cells, a precursor cell of platelets, was significantly reduced by treating the cells with $G$ proteincoupled receptor (GPCR) modulators, such as pertussis toxin or cholera toxin, or by using a global GPCR inhibitor. These findings suggest that GPCR signalling is involved in the PAGln cellular response.

Given that PAGln has a similar structure to catecholamines that are known to bind to adrenergic receptors, loss-of-function and gain-of-function studies were performed using both genetic and pharmacological approaches to determine whether adrenergic receptors were involved in PAGln-mediated cellular signalling. PAGln was shown to induce cellular responses via $\alpha_{2 A}, \alpha_{2 B}$ and $\beta_{2}$ adrenergic receptors, which are all present on human platelets.

Finally, PAGln exposure in the presence of propranolol, a $\beta$-adrenergic receptor antagonist (or $\beta$-blocker), attenuated PAGln-mediated platelet hyper-responsiveness. Similarly, the prothrombotic effects of PAGln were reversed with carvedilol treatment in mice compared with untreated controls.

"These findings substantially expand our knowledge of potential pathways through which the gut microbiome can contribute to the development of cardiovascular disease in humans," comments Hazen. "They also suggest that analyses of PAGln blood levels might be beneficial in helping to identify those at heightened cardiovascular risk who are otherwise not recognized by traditional cardiovascular risk factors."

Karina Huynh

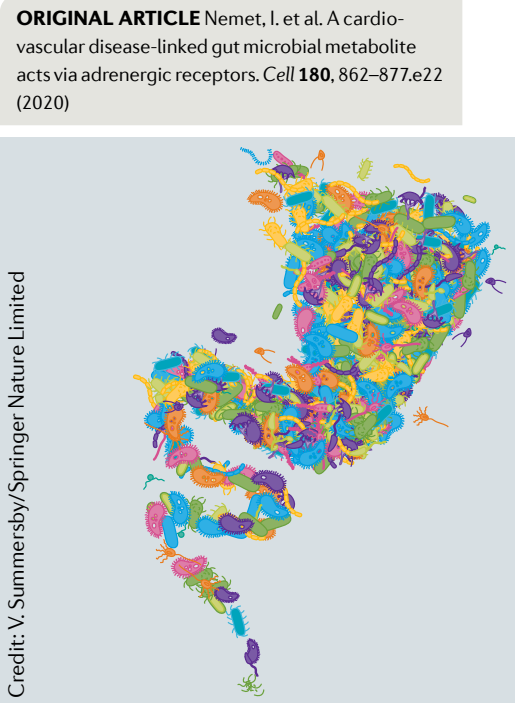

